\title{
New approaches to provide feedback from nuclear and covariance data adjustment for effective improvement of evaluated nuclear data files
}

\author{
Giuseppe Palmiotti ${ }^{1}$ a, Massimo Salvatores ${ }^{1}$, Mathieu Hursin ${ }^{2}$, Ivo Kodeli ${ }^{3}$, Fabrizio Gabrielli ${ }^{4}$, and Andrew Hummel ${ }^{1}$ \\ 1 Idaho National Laboratory, 2525 Fremont Ave., PO Box 1625, Idaho Falls, ID 83415-3860, USA \\ 2 Paul Scherrer Institut, Würenlingen, Switzerland \\ 3 Jožef Stefan Institute, Ljubljana, Slovenia \\ ${ }^{4}$ Karlsruhe Institute of Technology, Karlsruhe, Germany
}

\begin{abstract}
A critical examination of the role of uncertainty assessment, target accuracies, role of integral experiment for validation and, consequently, of data adjustments methods is underway since several years at OECD-NEA, the objective being to provide criteria and practical approaches to use effectively the results of sensitivity analyses and cross section adjustments for feedback to evaluators and experimentalists in order to improve without ambiguities the knowledge of neutron cross sections, uncertainties, and correlations to be used in a wide range of applications and to meet new requirements and constraints for innovative reactor and fuel cycle system design. An approach will be described that expands as much as possible the use in the adjustment procedure of selected integral experiments that provide information on "elementary" phenomena, on separated individual physics effects related to specific isotopes or on specific energy ranges. An application to a large experimental data base has been performed and the results are discussed in the perspective of new evaluation projects like the CIELO initiative.
\end{abstract}

\section{Introduction}

In the last decade there has been a growing research activity in the field of nuclear data for applications, due a growing awareness of a series of new challenges and possibly new paradigms in order to meet new requirements and constraints when approaching new reactor system design. The major driving forces have been:

- New reactor systems initiatives (like Generation IV), and, in parallel, proposals to meet waste management issues using innovative reactor systems as ADS or Fusion-Fission Hybrids.

- In both cases, it became clear at very early stages of research that the associated fuel cycle challenges were crucial and that in many cases their feasibility assessment could point out to drastic show stoppers.

- Also, during this decade, even with hesitations, mostly financing related, and even accounting for the impact of the Fukushima accident, a number of innovative projects, besides a revival of new "standard" power plants implementation, in particular in Asia, have been started in different areas, with the common purpose to facilitate a future more robust development of nuclear energy: the TerraPower TWR, the French ASTRID fast reactor, a number of SMR designs, a revival of interest for the Thorium cycle and molten salts reactors.
For all these initiatives, there is a common understanding of the need of improved and carefully validated calculation methods and data.

As far as nuclear data, a critical examination of the role of uncertainty assessment, target accuracies, role of integral experiment for validation and, consequently, of data adjustments methods is underway since several years in the frame of successive OECD-NEA WPEC subgroups. Subgroup 26 [1] was the starting point to promote data covariance development, to allow meaningful uncertainty analysis and target accuracy requirements; Subgroup 33 [2] has succeeded in providing a deeper understanding of nuclear data and associated covariance adjustment methods, the role of integral experiment uncertainties and of their application. The ongoing WPEC subgroup 39 [3] is intended to provide criteria and practical approaches to use effectively the results of sensitivity analyses and neutron cross section adjustments for feedback to evaluators and differential measurement experimentalists in order to improve without ambiguities the knowledge of neutron cross sections, uncertainties, and correlations to be used in a wide range of applications.

\section{A new approach using an ad-hoc enlarged integral data base}

An approach has been envisaged that expands as much as possible the use of selected integral experiments in the adjustment procedure that provide information on "elementary" phenomena, on separated individual physics effects related to specific isotopes or on specific energy

\footnotetext{
a e-mail: Giuseppe.Palmiotti@inl.gov
}

(C) The Authors, published by EDP Sciences. This is an Open Access article distributed under the terms of the Creative Commons Attribution License 4.0 (http://creativecommons.org/licenses/by/4.0/). 


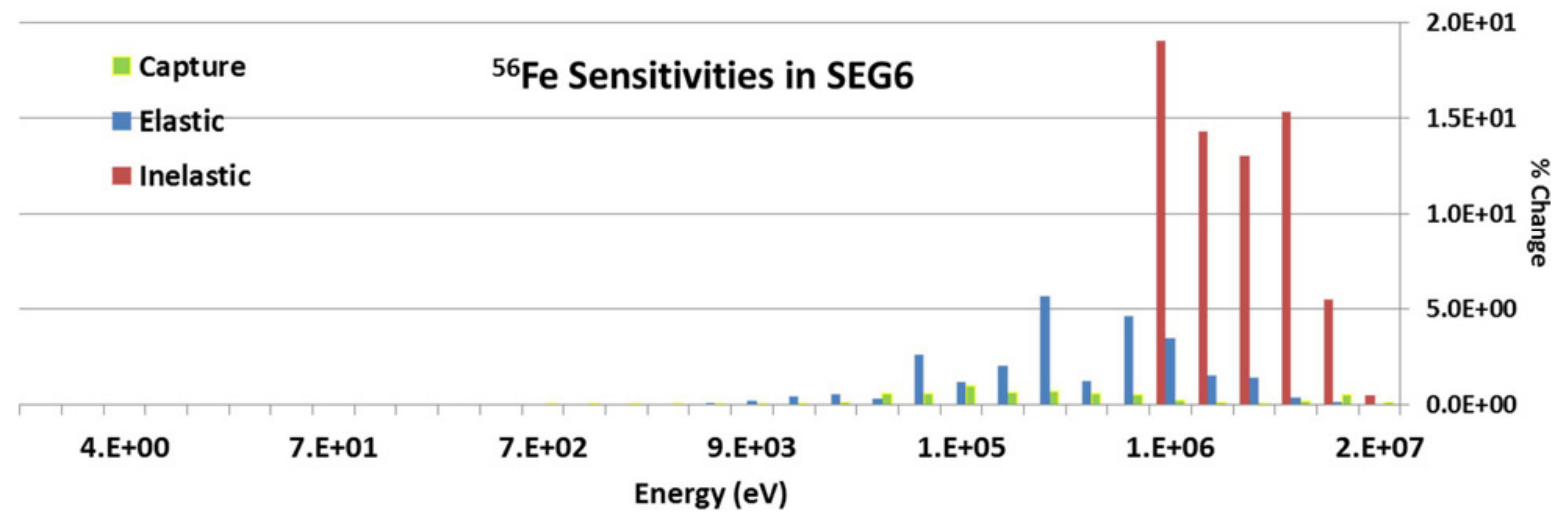

Figure 1. Examples of sensitivity profiles in SEG.

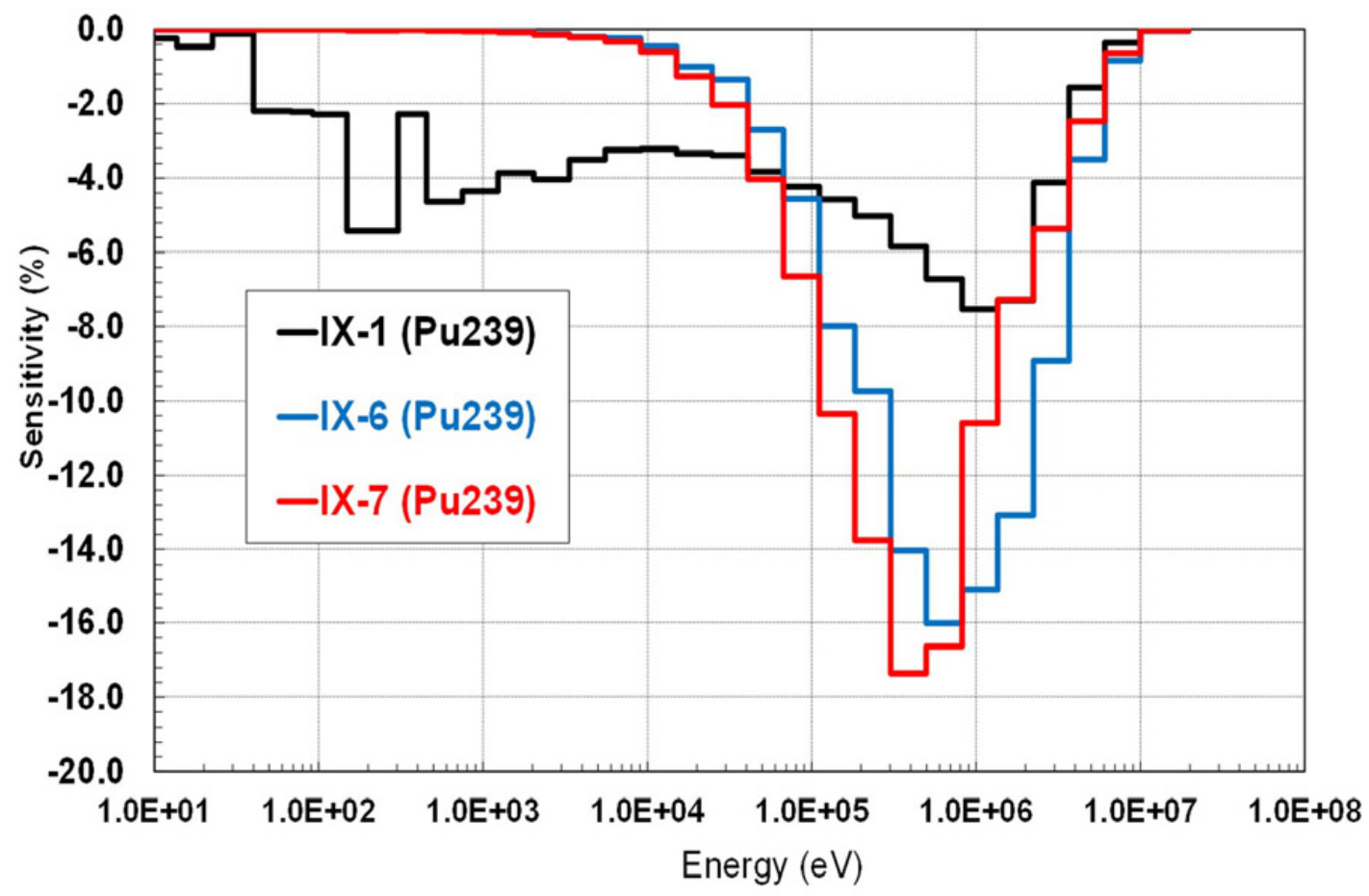

Figure 2. Examples of sensitivity profiles in FCA.

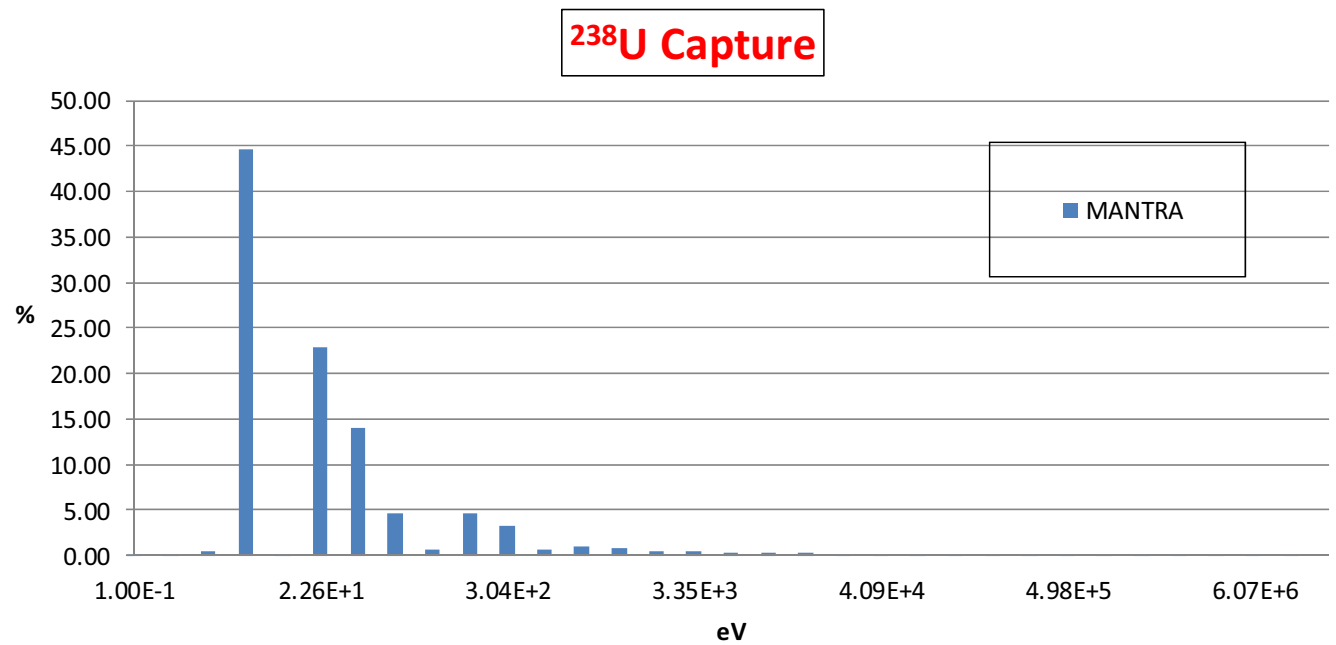

Figure 3. Examples of sensitivity profiles in MANTRA. 


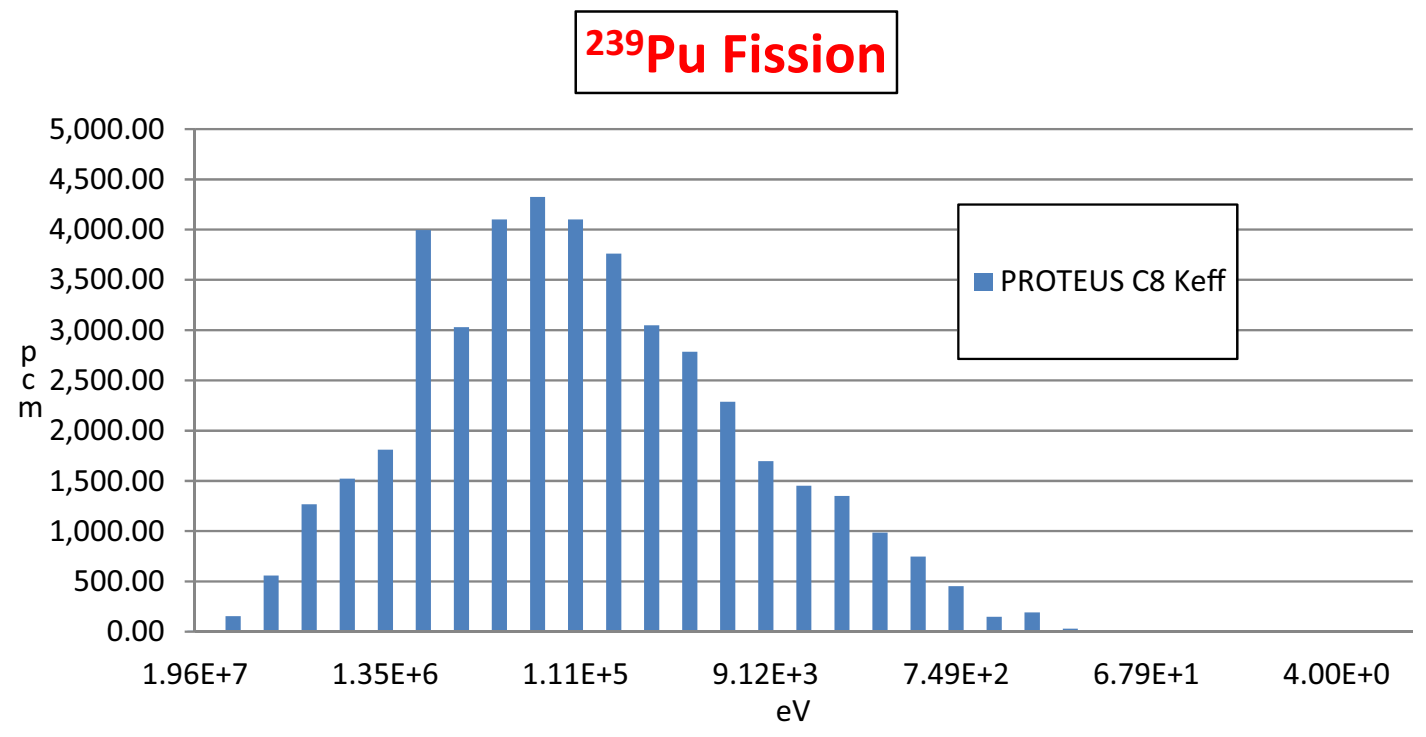

Figure 4. Examples of sensitivity profiles in PROTEUS.

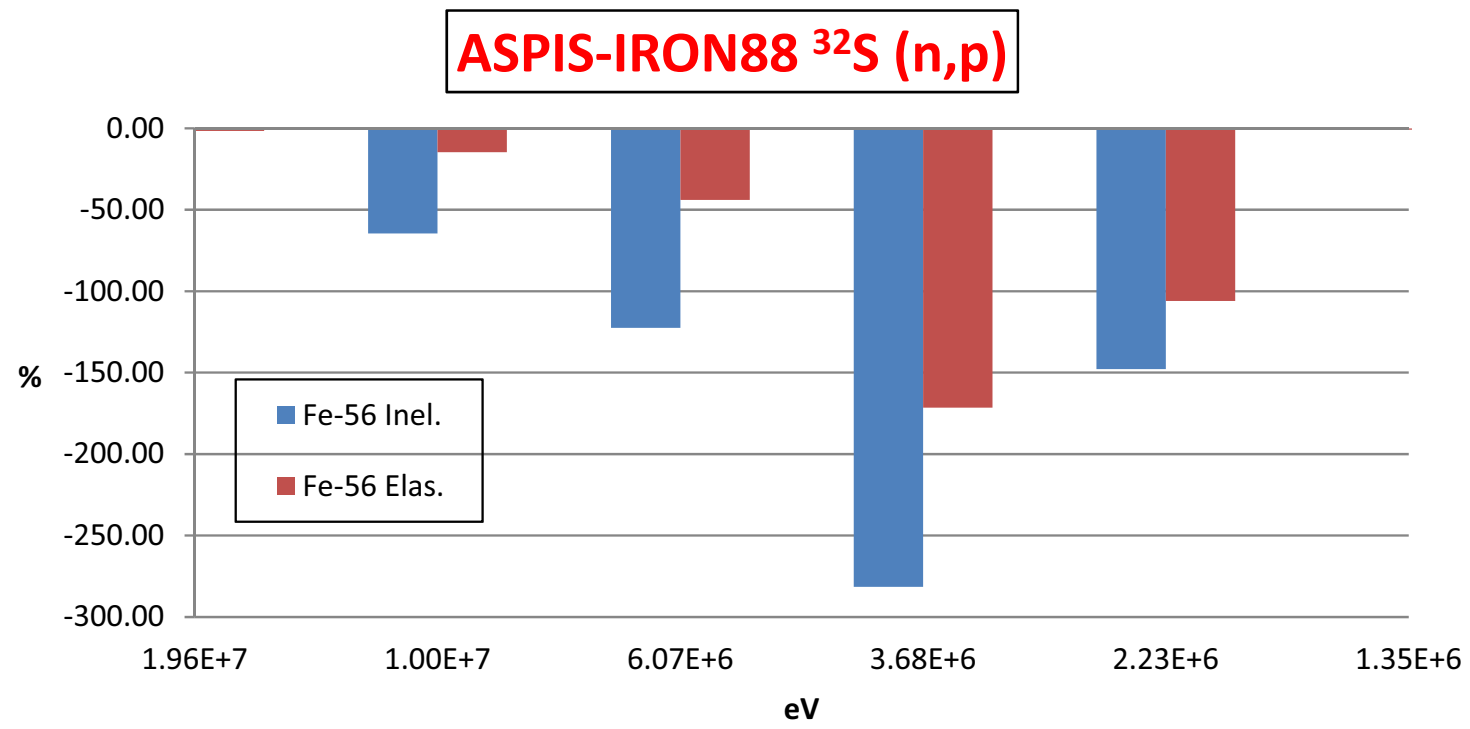

Figure 5. Examples of sensitivity profiles in ASPIS-IRON88.

ranges. In practice, in order to complement and support the new evaluation effort of the WPEC CIELO Subgroup [4], most of the elementary experiments that have been selected and added to the extended adjustment provide high sensitivities to neutron cross sections of the five priority CIELO isotopes: ${ }^{16} \mathrm{O},{ }^{56} \mathrm{Fe},{ }^{235} \mathrm{U},{ }^{238} \mathrm{U}$, and ${ }^{239} \mathrm{Pu}$.

In order to enlarge the "classical" set of integral experiments (criticality, reaction rates, and reactivity coefficients) in the fast energy range, the following more focused experiments are used:

Experiments providing selective information on inelastic, elastic, fission, and capture data (SEG experiments with ad-hoc tailored adjoint energy shape, [5]); FCA-IX experiments [6] with systematic variation of the spectrum hardness. Separated isotope sample irradiation experiments have been already widely used in past adjustments [7];

- Experiments with enhanced sensitivity to the actinide cross sections in the energy range $\leq 1 \mathrm{keV}: \mathrm{k}_{\infty}$, reaction rates, void reactivity measurements performed at the PROTEUS facility in the frame of a High Conversion LWR validation program, [8];

- Experiments with enhanced capture data sensitivity in the range from few hundred $\mathrm{eV}$ to $1 \mathrm{eV}$ (MANTRA irradiation experiments at ATR with appropriate filters to tune the spectrum at the irradiation position, [9]);

- Experiments providing specific feedbacks on elastic and inelastic cross sections of structural materials (ASPIS-IRON88 neutron propagation experiment in $\mathrm{Fe},[10])$.

In order to understand the potential role of these new experiments, sensitivity analysis has been performed for each new integral parameter. Examples are given in Figs. 1 to 5.

As expected, the sensitivity profiles confirm the potential role of these experiments for specific reactions and energy regions and can help to discriminate between, e.g., inelastic and capture cross sections. 
Table 1 summarizes the full set of experiment (124) that have been used (facility, configuration, type of experiments performed). The description and/or the references of the experiments used in past adjustments can be found in [7]. New experiments are indicated with a* symbol.

\section{Statistical adjustment}

The statistical adjustment was performed using the method described in [2] and widely used in previous studies. This method has been shown to be equivalent to similar adjustment method, as shown by the extensive comparison of [2].

The multigroup neutron cross section adjustment has been carried out using ENDF/B-VII.0 data files and COMMARA 2.0 covariance matrix [11], plus elastic anisotropy from JENDL-4 for the CIELO isotopes.

The initial set of more than 200 integral experimental quantities has been analyzed by using the best calculation tools available, mostly Monte Carlo, as described in [7], in order to provide $\mathrm{C} / \mathrm{E}$ and associated calculation and experimental uncertainties and correlations. The correlations were obtained using the approach described in [7].

A larger initial set of data was reduced to 124 experimental values based on several considerations, i.e., duplications, sensitivities, inconsistency after adjustment, experimental uncertainties, etc..

A total of 45 isotopes including major and minor actinides, fission products (FP), structural, coolant, and light isotopes were adjusted.

A 33 energy group structure was adopted (shown in Table 2) and sensitivity coefficients were calculated. Generalized Perturbation Theory (GPT) was used for static integral parameters and Time Dependent Perturbation Theory (TDPT) for isotope build up.

The result of the adjustment is a strong reduction of the original $(\mathrm{E}-\mathrm{C}) / \mathrm{C}$ values, associated to cross section adjustments (see later on) with a generalized reduction of the initial cross section uncertainties. The statistical $\chi^{2}(=0.982)$ tests indicate an overall reliability of the adjustment. The most significant $(\mathrm{E}-\mathrm{C}) / \mathrm{C}$ reductions are show in Table 3 and Table 4 (devoted to fission products related experiments).

The reductions of the $(\mathrm{E}-\mathrm{C}) / \mathrm{C}$ discrepancy are related to specific nuclear data changes. A few examples of the major contributions in selected cases are given in Tables 5-8. This type of analysis allow understanding the role of individual experiments and, hopefully, to underline their complementary.

The data in Table 5 show the contributions of nuclear data to the reduction of $\mathrm{k}_{\mathrm{eff}}(\mathrm{E}-\mathrm{C}) / \mathrm{C}$ depending on the core major constituents and the type of neutron spectrum. In fact, ZPR9-34 is a U/Fe assembly with SS reflector while ZPR3-53 is a Pu/C/SS assembly with U blanket. However, in both cases the adjustment of the fission spectra plays an important role.

Table 6 shows the results for two experiments strongly dependent on Fe-56 data. Both have large sensitivities to elastic and inelastic cross sections but in complementary way, the ASPIS-IRON88 $\mathrm{S}(\mathrm{n}, \mathrm{p})$ neutron propagation detection experiment being more sensitive to higher energy data with respect to the SEG6 Fe sample reactivity measurement
Table 1. Experiments used in the adjustment.

\begin{tabular}{|c|c|c|c|}
\hline Facility & Experiment & $\begin{array}{c}\text { Type of } \\
\text { experiment }\end{array}$ & $\begin{array}{l}\text { Number } \\
\text { of } \\
\text { experim. }\end{array}$ \\
\hline $\begin{array}{l}\text { LANL Small } \\
\text { criticals }\end{array}$ & $\begin{array}{c}\text { GODIVA, } \\
\text { JEZEBEL } \\
\text { BIGTEN, Np } \\
\text { SPHERE }\end{array}$ & $\begin{array}{l}\text { Keff, reaction } \\
\text { rate ratios }\end{array}$ & 17 \\
\hline ZPR/ZPPR & $\begin{array}{c}\text { ZPR6/7 ; } \\
\text { ZPR3/53 and } \\
54 \\
\text { ZPR9-34 } \\
\text { ZPPR-9 } \\
\text { ZPPR-10 } \\
\text { ZPPR-15 }\end{array}$ & $\begin{array}{c}\text { Keff; void } \\
\text { reactivity, } \\
\text { central control } \\
\text { rod } \\
\text { Fission and } \\
\text { capture rate } \\
\text { ratios major } \\
\text { actinides } \\
\text { Flux radial } \\
\text { distribution } \\
\text { slopes } \\
\end{array}$ & 19 \\
\hline MASURCA & $\begin{array}{l}\text { CIRANO } \\
\text { COSMO }\end{array}$ & $\begin{array}{l}\mathrm{K}_{\text {eff; }} \text {, reaction } \\
\text { rate ratios } \\
\text { (major and } \\
\text { minor } \\
\text { actinides) }\end{array}$ & 9 \\
\hline PHENIX & $\begin{array}{l}\text { PROFIL1; } \\
\text { PROFIL2 ; } \\
\text { TRAPU }\end{array}$ & $\begin{array}{c}\text { Separated } \\
\text { isotope irradiati } \\
\text { ons: major, } \\
\text { minor } \\
\text { actinides, } \\
\text { fission } \\
\text { products. } \\
\text { Variable } \\
\text { actinide content } \\
\text { pins } \\
\end{array}$ & 36 \\
\hline ATR & MANTRA* & $\begin{array}{c}\text { Separated } \\
\text { isotope irradiati } \\
\text { ons: major and } \\
\text { minor actinide } \\
\text { isotopes }\end{array}$ & 3 \\
\hline PROTEUS & $\begin{array}{l}\text { HCLWR } \\
\text { phase II * }\end{array}$ & $\begin{array}{l}\mathrm{K}_{\infty} ; \text { void } \\
\text { reactivity; } \\
\text { reaction rate } \\
\text { ratios }\end{array}$ & 8 \\
\hline SEG & $\begin{array}{c}\text { SEG5 ; SEG6 } \\
*\end{array}$ & $\begin{array}{l}\text { Sample } \\
\text { reactivity } \\
\text { worths }\end{array}$ & 2 \\
\hline ASPIS & IRON88 * & $\begin{array}{c}\text { Foil detectors } \\
\text { at several radial } \\
\text { positions }\end{array}$ & 11 \\
\hline JOYO & & $K_{\text {eff }}$ & 1 \\
\hline FCA & FCA-IX* & $\begin{array}{l}\text { Reaction rate } \\
\text { ratios }\end{array}$ & 18 \\
\hline
\end{tabular}

Table 2. 33 energy group structure (eV). Lower energy boundary $1.110^{-4} \mathrm{eV}$.

\begin{tabular}{|c|c|c|c|c|c|}
\hline Group & Up Ener. & Group & Up Ener. & Group & Up Ener. \\
\hline 1 & $1.9610^{7}$ & 12 & $6.7410^{4}$ & 23 & $3.0410^{2}$ \\
\hline 2 & $1.0010^{7}$ & 13 & $4.0910^{4}$ & 24 & $1.4910^{2}$ \\
\hline 3 & $6.0710^{6}$ & 14 & $2.4810^{4}$ & 25 & $9.1710^{1}$ \\
\hline 4 & $3.6810^{6}$ & 15 & $1.5010^{4}$ & 26 & $6.7910^{1}$ \\
\hline 5 & $2.2310^{6}$ & 16 & $9.1210^{3}$ & 27 & $4.0210^{1}$ \\
\hline 6 & $1.3510^{6}$ & 17 & $5.5310^{3}$ & 28 & $2.2610^{1}$ \\
\hline 7 & $8.2110^{5}$ & 18 & $3.3510^{3}$ & 29 & $1.3710^{1}$ \\
\hline 8 & $4.9810^{5}$ & 19 & $2.0310^{3}$ & 30 & $8.3210^{0}$ \\
\hline 9 & $3.0210^{5}$ & 20 & $1.2310^{3}$ & 31 & $4.0010^{0}$ \\
\hline 10 & $1.8310^{5}$ & 21 & $7.4910^{2}$ & 32 & $5.4010^{-1}$ \\
\hline 11 & $1.1110^{5}$ & 22 & $4.5410^{2}$ & 33 & $1.0010^{-1}$ \\
\hline
\end{tabular}


Table 3. Most significant (E-C)/C reduction for selected experiments.

\begin{tabular}{|c|c|c|c|c|c|}
\hline Experiment & $\begin{array}{c}\text { Initial } \\
(\mathrm{E}-\mathrm{C}) / \mathrm{C} \\
\%\end{array}$ & $\begin{array}{c}\text { Final } \\
(\mathrm{E}-\mathrm{C}) / \mathrm{C} \\
\%\end{array}$ & Experiment & $\begin{array}{c}\text { Initial } \\
(\mathrm{E}-\mathrm{C}) / \mathrm{C} \\
\%\end{array}$ & $\begin{array}{c}\text { Final } \\
(\mathrm{E}-\mathrm{C}) / \mathrm{C} \\
\%\end{array}$ \\
\hline ZPR3-54 $\mathrm{K}_{\mathrm{eff}}$ & -1.188 & 0.017 & BIGTEN F28/F25 & 5.6 & 0.6 \\
\hline ZPR3-53 $\mathrm{K}_{\mathrm{eff}}$ & -0.915 & -0.028 & BIGTEN F37/F25 & 3.4 & -0.4 \\
\hline ZPR9-34 Keff & -0.874 & -0.007 & BIGTEN F49/F25 & 2.6 & 0.9 \\
\hline PROTEUS Void Coef. & 480.0 & 2.3 & COSMO F28/F25 & 1.626 & -0.76 \\
\hline PROTEUS-C8 keff & 3.950 & 1.881 & FCA-IX-7 F53/F49 & 6.7 & -2.2 \\
\hline PROTEUS-C8 C42/F49 & -10.7 & 0.07 & FCA-IX-1 F53/F49 & 8.7 & 0.0 \\
\hline PROTEUS-C8 F25/F49 & -2.2 & -0.4 & FCA-IX-6 F53/F49 & 10.2 & 2.8 \\
\hline TRAPU Cm243 Build-up & 107.0 & 0.07 & FCA-IX-7 F51/F49 & 7.0 & 1.5 \\
\hline PROFIL1 ${ }^{238} \mathrm{Pu}$ in ${ }^{239} \mathrm{Pu}$ sample & 32.8 & 8.3 & FCA-IX-1 F51/F49 & 5.5 & 0.5 \\
\hline PROFIL2 ${ }^{245} \mathrm{Cm}$ in ${ }^{244} \mathrm{Cm}$ sample & -8.7 & 1.9 & FCA-IX-6 F51/F49 & 7.6 & 4.1 \\
\hline PROFIL1 ${ }^{243} \mathrm{Am}$ in ${ }^{242} \mathrm{Pu}$ sample & -5.6 & 0.8 & FCA-IX-7 F42/F49 & -4.5 & -2.2 \\
\hline PROFIL1 ${ }^{240} \mathrm{Pu}$ in ${ }^{239} \mathrm{Pu}$ sample & 10.3 & 5.5 & FCA-IX-6 F42/F49 & -3.5 & 0.6 \\
\hline PROFIL $2{ }^{238} \mathrm{Pu}$ in ${ }^{237} \mathrm{~Np}$ sample & 6.7 & 3.3 & ASPIS-FE-88 Al $(\mathrm{n}, \alpha) \mathrm{A} 7$ & -25.9 & 1.6 \\
\hline Np Sphere $K_{\text {eff }}$ & 0.562 & 0.247 & ASPIS-FE-88 S(n,p) A12 & 6.5 & 0.2 \\
\hline SEG6 Fe sample & 5.5 & 3.0 & ASPIS-FE-88 Rh(n, n')A14 & -9.0 & 1.1 \\
\hline Godiva F28/F25 & 4.7 & 0.5 & ASPIS-FE-88 Rh(n, n')A7 & -5.1 & 1.5 \\
\hline
\end{tabular}

Table 4. Most significant (E-C)/C reduction for fission product related experiments.

\begin{tabular}{|c|c|c|}
\hline Experiment & $\begin{array}{c}\text { Initial (E-C)/C } \\
\%\end{array}$ & $\begin{array}{c}\text { Final (E-C)/C } \\
\%\end{array}$ \\
\hline $\begin{array}{c}\text { PROFIL1 }{ }^{106} \mathrm{Pd} \text { in } \\
{ }^{105} \mathrm{Pd} \text { sample }\end{array}$ & 17.9 & 4.8 \\
\hline $\begin{array}{c}\text { PROFIL1 }{ }^{102} \mathrm{Ru} \text { in } \\
{ }^{101} \mathrm{Ru} \text { sample }\end{array}$ & -9.4 & 3.4 \\
\hline $\begin{array}{c}\text { PROFIL2 }{ }^{152} \mathrm{Sm} \text { in } \\
{ }^{151} \mathrm{Sm} \text { sample }\end{array}$ & -10.0 & 1.7 \\
\hline $\begin{array}{c}\text { PROFIL1 }{ }^{134} \mathrm{Cs} \text { in } \\
{ }^{133} \mathrm{Cs} \text { sample }\end{array}$ & 13.7 & 4.3 \\
\hline $\begin{array}{c}\text { PROFIL2 }{ }^{154} \mathrm{Eu} \text { in } \\
{ }^{153} \mathrm{Eu} \text { sample }\end{array}$ & 9.7 & 1.6 \\
\hline $\begin{array}{c}\text { PROFIL2 }{ }^{107} \mathrm{Pd} \text { in } \\
{ }^{106} \mathrm{Pd} \text { sample }\end{array}$ & 6.7 & 1.7 \\
\hline $\begin{array}{c}\text { PROFIL1 }{ }^{96} \mathrm{Mo} \text { in } \\
{ }^{95} \mathrm{Mo} \text { sample }\end{array}$ & -3.2 & 1.2 \\
\hline
\end{tabular}

Finally, Table 7 indicates the improvement of the reaction rate ratio $\mathrm{F} 53 / \mathrm{F} 49$ obtained in the FCA IX experiments: the harder spectrum in the FCA IX-6 experiment show the effects of the ${ }^{243}$ Am fission cross section variations and some spectrum modification effects associate to inelastic cross section variations, while the FCA IX-1 softer spectrum experiment shows some effects related to low energy ${ }^{239} \mathrm{Pu}$ fission cross section variation. Also in the case of the FCA experiments, adjustments of the fission spectra are significant.

\section{Feedback on nuclear data}

As far as cross section adjustments, the most significant trends are indicated below and shown in Figs. 6-13:

- ${ }^{16}$ O: Significant elastic cross section decrease ( $\sim 6 \%$ and outside current standard deviation) and some impact on $\mathrm{P}_{1}$ scattering.

- ${ }^{56} \mathrm{Fe}$ : Systematic increase of capture cross section (higher at low energy) at $1.23 \mathrm{keV}$ resonance a $5 \%-6 \%$ increase is observed. No major change in inelastic and some change in $\mathrm{P}_{1}$.
- ${ }^{235} \mathrm{U}$ : little changes in capture. Significant decrease of $\chi$ below $500 \mathrm{KeV} . \mathrm{P}_{1}$ elastic decreases between 800 and $100 \mathrm{KeV}$. Systematic decrease of inelastic (with respect to ENDF/B-VII.0).

- ${ }^{238} \mathrm{U}$ : decrease of capture $(\sim 4 \%$ average from $25 \mathrm{KeV}$ to $1 \mathrm{KeV}$ ) of inelastic $\sim 5-10 \%$. Change in shape of $\chi$. Significant increase of the $\mathrm{P}_{1}$ component (200 KeV to $5 \mathrm{KeV})$.

- ${ }^{239} \mathrm{Pu}$ : Significant increase ( $\left.10 \%\right)$ of capture from $10 \mathrm{KeV}$ to $1 \mathrm{KeV}$; some also at thermal energies. Significant decrease of fission (average $\sim 4 \%$ ) below $\sim 1 \mathrm{KeV}$ (also at thermal energies). Change in shape of inelastic (significant). No significant change in $\chi$. Large change of $n, 2 n(\sim+30 \%$ from 10 to $6 \mathrm{MeV}$ ).

An important feature of the adjustment is the generalized reduction of uncertainties (see e.g., Figs. 14 and 15) for standard deviations. Regarding the significant reduction in uncertainty due to also the introduction of negative cross correlations, Table 8 show the uncertainties for some selected experiments before and after adjustments.

In general, fission spectrum adjustments (not attempted before) have a very large impact in the adjustment.

For small critical experiments (JEZEBEL, FLATTOP, BIGTEN) there are many compensations among $\chi$, fission, and inelastic data for the major actinides. Similar compensations are also found in large critical experiments (JOYO, ZPR-6/7, ZPR-9/34, ZPR-3/53 and /54) with some contribution coming from the $238 \mathrm{U}$ capture too.

As far as the new selected experiments, it has been found significant effects: a) FCA experiments are sensitive to inelastic cross sections and fission spectrum, which allow a change in spectrum; b) The reduction of the significant $\mathrm{C} / \mathrm{E}$ discrepancy on the PROTEUS void reactivity is due to many competing effects: in particular, the low energy data of ${ }^{239} \mathrm{Pu}$ and ${ }^{241} \mathrm{Pu}$ play an important role; c) adjoint tailored experiments show clearly the role of ${ }^{238} \mathrm{U}$ inelastic (SEG6 steep adjoint) and ${ }^{56} \mathrm{Fe}$ elastic and inelastic (SEG5-6); d) the ASPIS-IRON88 experiments 
Table 5. Contribution to parameter change by adjusted data (\%) to ZPR9-34 and ZPR3-53 experiments.

\begin{tabular}{|c|c|c|c|c|c|c|c|}
\hline \multicolumn{4}{|c|}{ ZPR9-34 keff } & \multicolumn{4}{|c|}{ ZPR3-53 keff } \\
\hline Isotope & Reaction & $\begin{array}{l}\text { Energy } \\
\text { Group }\end{array}$ & $\begin{array}{c}\text { Contribution } \\
\%\end{array}$ & Isotope & Reaction & $\begin{array}{l}\text { Energy } \\
\text { Group }\end{array}$ & $\begin{array}{c}\text { Contribution } \\
\%\end{array}$ \\
\hline \multirow{9}{*}{${ }^{235} \mathrm{U}$} & \multirow{9}{*}{$\chi$} & 3 & 0.37 & \multirow{9}{*}{${ }^{239} \mathrm{Pu}$} & \multirow{5}{*}{$\chi$} & 3 & -0.264 \\
\hline & & 4 & 0.95 & & & 4 & -0.144 \\
\hline & & 5 & 0.25 & & & 5 & 0.140 \\
\hline & & 6 & -0.22 & & & 6 & 0.191 \\
\hline & & 7 & -0.34 & & & 7 & 0.115 \\
\hline & & 8 & -0.38 & & \multirow{2}{*}{ fission } & 20 & -0.087 \\
\hline & & 9 & -0.27 & & & 21 & -0.083 \\
\hline & & 10 & -015 & & \multirow{2}{*}{ capture } & 16 & -0.091 \\
\hline & & 11 & -0.08 & & & 17 & -0.113 \\
\hline \multirow{6}{*}{${ }^{56} \mathrm{Fe}$} & \multirow{2}{*}{ capture } & 7 & -0.07 & \multirow{6}{*}{${ }^{238} \mathrm{U}$} & \multirow{6}{*}{$\chi$} & 2 & -0.247 \\
\hline & & 20 & -0.12 & & & 3 & -0.329 \\
\hline & \multirow{4}{*}{ elastic } & 7 & 0.10 & & & 4 & -0.088 \\
\hline & & 8 & 0.11 & & & 5 & 0.131 \\
\hline & & 9 & 0.10 & & & 6 & 0.139 \\
\hline & & 10 & 0.10 & & & 7 & 0.098 \\
\hline \multicolumn{2}{|c|}{ Total } & \multicolumn{2}{|c|}{$-0.867 \%$} & \multicolumn{2}{|c|}{ Total } & \multicolumn{2}{|c|}{$-0.887 \%$} \\
\hline
\end{tabular}

Table 6. Contribution to parameter change by adjusted data (\%) to SEG6 and ASPIS-88 experiments.

\begin{tabular}{|c|c|c|c|c|c|c|c|}
\hline \multicolumn{4}{|c|}{ SEG6 Fe sample } & \multicolumn{4}{|c|}{ ASPIS-88 S(n,p) A14 } \\
\hline Isotope & Reaction & $\begin{array}{l}\text { Energy } \\
\text { Group }\end{array}$ & $\begin{array}{c}\text { Contribution } \\
\%\end{array}$ & Isotope & Reaction & $\begin{array}{l}\text { Energy } \\
\text { Group }\end{array}$ & $\begin{array}{c}\text { Contribution } \\
\%\end{array}$ \\
\hline \multirow{15}{*}{${ }^{56} \mathrm{Fe}$} & \multirow{9}{*}{ elastic } & 5 & -0.13 & \multirow{10}{*}{${ }^{56} \mathrm{Fe}$} & \multirow{4}{*}{ elastic } & 2 & 0.14 \\
\hline & & 6 & -0.06 & & & 3 & 1.65 \\
\hline & & 7 & 0.17 & & & 4 & 8.00 \\
\hline & & 8 & 0.11 & & & 5 & 7.28 \\
\hline & & 9 & 0.10 & & P1 elastic & 3 & 0.08 \\
\hline & & 10 & 0.09 & & \multirow{5}{*}{ inelastic } & 1 & -0.08 \\
\hline & & 11 & 0.13 & & & 2 & -0.52 \\
\hline & & 12 & 0.12 & & & 3 & -2.33 \\
\hline & & 13 & 0.12 & & & 4 & -2.48 \\
\hline & \multirow{2}{*}{ capture } & 7 & 0.10 & & & 5 & -6.71 \\
\hline & & 8 & 0.06 & \multirow{5}{*}{${ }^{235} \mathrm{U}$} & \multirow{5}{*}{$\chi$} & 1 & -0.61 \\
\hline & \multirow{4}{*}{ inelastic } & 3 & 0.24 & & & 2 & -0.30 \\
\hline & & 4 & 0.12 & & & 3 & 1.08 \\
\hline & & 5 & 0.68 & & & 4 & 1.90 \\
\hline & & 6 & 0.40 & & & 5 & 0.10 \\
\hline \multicolumn{2}{|c|}{ Total change } & \multicolumn{2}{|c|}{$2.4 \%$} & \multicolumn{2}{|c|}{ Total change } & \multicolumn{2}{|c|}{$7.4 \%$} \\
\hline
\end{tabular}

Table 7. Contribution to parameter change by adjusted data (\%) to FCA IX-6 and FCA-6 experiments.

\begin{tabular}{|c|c|c|c|c|c|c|c|}
\hline \multicolumn{4}{|c|}{ FCA IX-6 F53/F49 } & \multicolumn{4}{|c|}{ FCA IX-1 F53/F49 } \\
\hline Isotope & Reaction & $\begin{array}{l}\text { Energy } \\
\text { Group }\end{array}$ & $\begin{array}{c}\text { Contribution } \\
\%\end{array}$ & Isotope & Reaction & $\begin{array}{l}\text { Energy } \\
\text { Group }\end{array}$ & $\begin{array}{c}\text { Contribution } \\
\%\end{array}$ \\
\hline \multirow{5}{*}{${ }^{243} \mathrm{Am}$} & \multirow{5}{*}{ fission } & 2 & 0.16 & \multirow{5}{*}{${ }^{243} \mathrm{Am}$} & \multirow{5}{*}{ fission } & 2 & 0.13 \\
\hline & & 3 & 0.65 & & & 3 & 0.57 \\
\hline & & 4 & 1.62 & & & 4 & 1.47 \\
\hline & & 5 & 1.97 & & & 5 & 2.17 \\
\hline & & 6 & 0.78 & & & 6 & 0.75 \\
\hline \multirow{8}{*}{${ }^{235} \mathrm{U}$} & \multirow{5}{*}{$\chi$} & 3 & 0.16 & \multirow{6}{*}{${ }^{235} \mathrm{U}$} & \multirow{6}{*}{$\chi$} & 3 & 0.33 \\
\hline & & 4 & 0.47 & & & 4 & 0.68 \\
\hline & & 7 & 0.28 & & & 7 & 0.33 \\
\hline & & 8 & 0.36 & & & 8 & 0.39 \\
\hline & & 9 & 0.23 & & & 9 & 0.26 \\
\hline & \multirow{3}{*}{ inelastic } & 4 & 0.16 & & & 10 & 0.14 \\
\hline & & 5 & 0.21 & \multirow{4}{*}{${ }^{239} \mathrm{Pu}$} & \multirow{4}{*}{ fission } & 19 & 0.15 \\
\hline & & 6 & 0.21 & & & 20 & 0.17 \\
\hline${ }^{238} \mathrm{U}$ & inelastic & 6 & 0.22 & & & 21 & 0.17 \\
\hline${ }^{56} \mathrm{Fe}$ & inelastic & 5 & -0.15 & & & 23 & 0.22 \\
\hline \multicolumn{2}{|c|}{ Total change } & \multicolumn{2}{|c|}{$7.3 \%$} & \multicolumn{2}{|c|}{ Total change } & \multicolumn{2}{|c|}{$8.7 \%$} \\
\hline
\end{tabular}


Table 8. Uncertainty reduction for selected experiments.

\begin{tabular}{|c|c|c|c|c|c|}
\hline Experiment & $\begin{array}{c}\text { Initial } \\
\text { Uncertainty } \\
\%\end{array}$ & $\begin{array}{c}\text { Final } \\
\text { Uncertainty } \\
\%\end{array}$ & Experiment & $\begin{array}{c}\text { Initial } \\
\text { Uncertainty } \\
\%\end{array}$ & $\begin{array}{c}\text { Final } \\
\text { Uncertainty } \\
\%\end{array}$ \\
\hline ZPR3-54 $\mathrm{K}_{\text {eff }}$ & 1.092 & 0.149 & BIGTEN F28/F25 & 13.62 & 0.81 \\
\hline ZPR3-53 K eff & 0.760 & 0.095 & BIGTEN F37/F25 & 7.71 & 0.95 \\
\hline ZPR9-34 Keff & 2.433 & 0.108 & BIGTEN F49/F25 & 1.024 & 0.39 \\
\hline PROTEUS Void Coef. & 115.9 & 6.411 & COSMO F28/F25 & 6.0 & 0.86 \\
\hline PROTEUS-C8 keff & 2.110 & 0.227 & FCA-IX-7 F53/F49 & 11.62 & 1.5 \\
\hline PROTEUS-C8 C42/F49 & 19.11 & 2.312 & FCA-IX-1 F53/F49 & 9.19 & 1.5 \\
\hline PROTEUS-C8 F25/F49 & 1.051 & 0.424 & FCA-IX-6 F53/F49 & 9.08 & 1.5 \\
\hline TRAPU Cm243 Build-up & 49.19 & 3.64 & FCA-IX-7 F51/F49 & 7.40 & 1.15 \\
\hline PROFIL1 ${ }^{238} \mathrm{Pu}$ in ${ }^{239} \mathrm{Pu}$ sample & 16.43 & 7.59 & FCA-IX-1 F51/F49 & 3.40 & 1.17 \\
\hline PROFIL2 ${ }^{245} \mathrm{Cm}$ in ${ }^{244} \mathrm{Cm}$ sample & 33.42 & 2.16 & FCA-IX-6 F51/F49 & 2.63 & 1.06 \\
\hline PROFIL1 ${ }^{243} \mathrm{Am}$ in ${ }^{242} \mathrm{Pu}$ sample & 17.75 & 2.20 & FCA-IX-7 F42/F49 & 4.93 & 1.23 \\
\hline PROFIL1 ${ }^{240} \mathrm{Pu}$ in ${ }^{239} \mathrm{Pu}$ sample & 4.66 & 1.33 & FCA-IX-6 F42/F49 & 4.42 & 1.14 \\
\hline PROFIL22 ${ }^{238} \mathrm{Pu}$ in ${ }^{237} \mathrm{~Np}$ sample & 4.20 & 2.27 & ASPIS-FE-88 Al $(\mathrm{n}, \alpha)$ A7 & 62.66 & 5.11 \\
\hline Np Sphere K eff & 1.028 & 0.287 & ASPIS-FE-88 S(n,p) A12 & 20.42 & 4.98 \\
\hline SEG6 Fe sample & 4.37 & 1.51 & ASPIS-FE-88 Rh(n, $\left.\mathrm{n}^{\prime}\right)$ A14 & 22.79 & 4.01 \\
\hline Godiva F28/F25 & 4.43 & 1.06 & ASPIS-FE-88 Rh(n, n')A7 & 17.61 & 3.92 \\
\hline
\end{tabular}

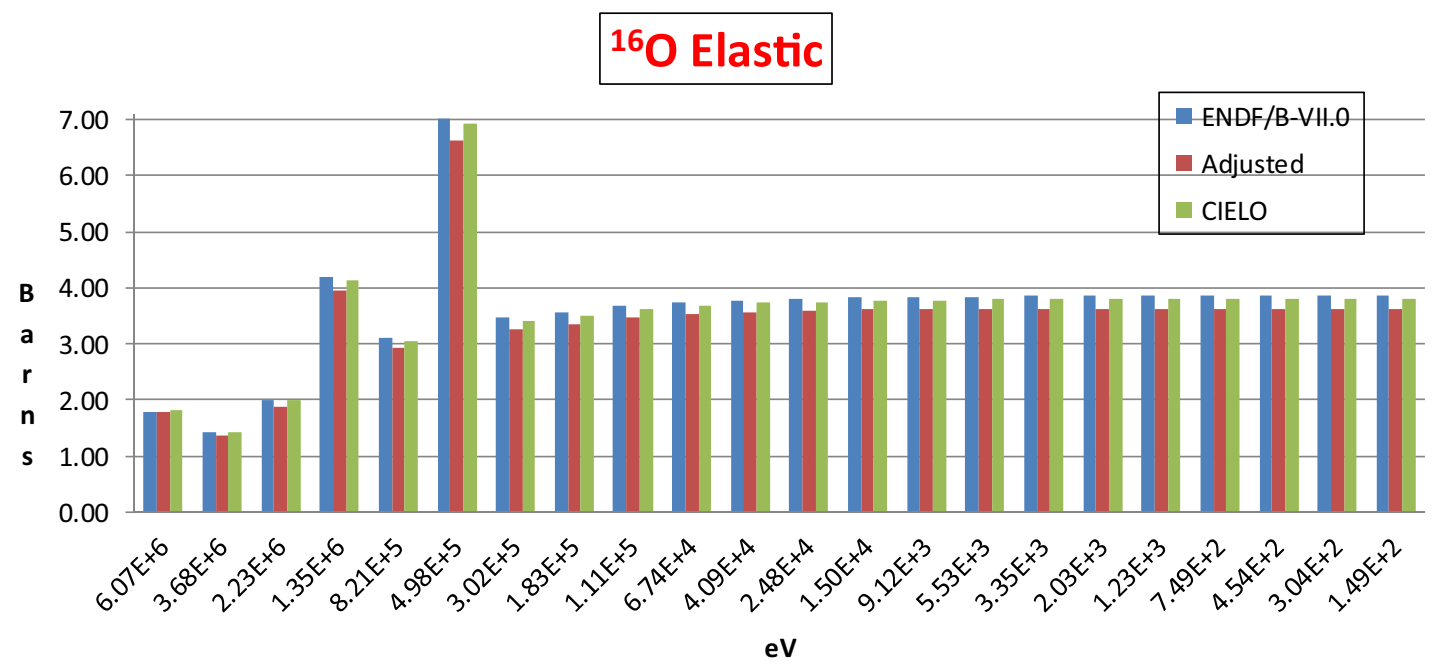

Figure 6. Examples of adjusted cross sections: ${ }^{16} \mathrm{O}$ elastic.

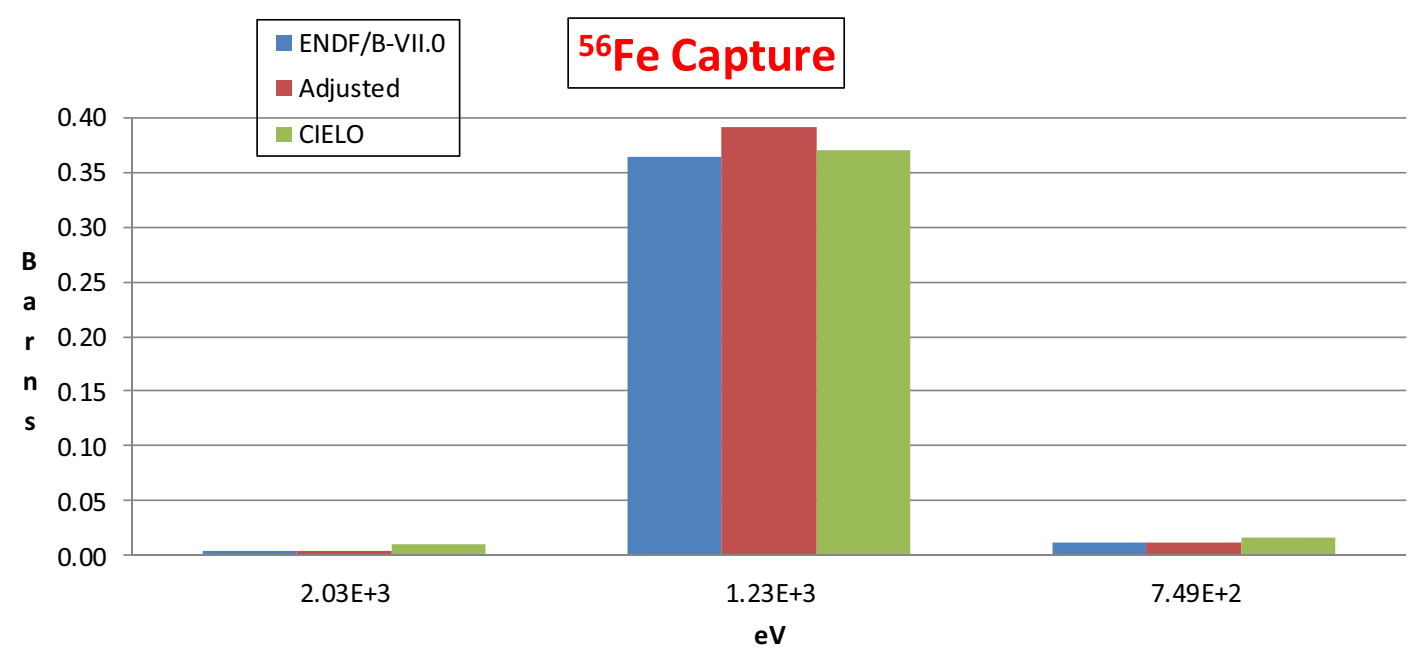

Figure 7. Examples of adjusted cross sections: ${ }^{56} \mathrm{Fe}$ capture. 


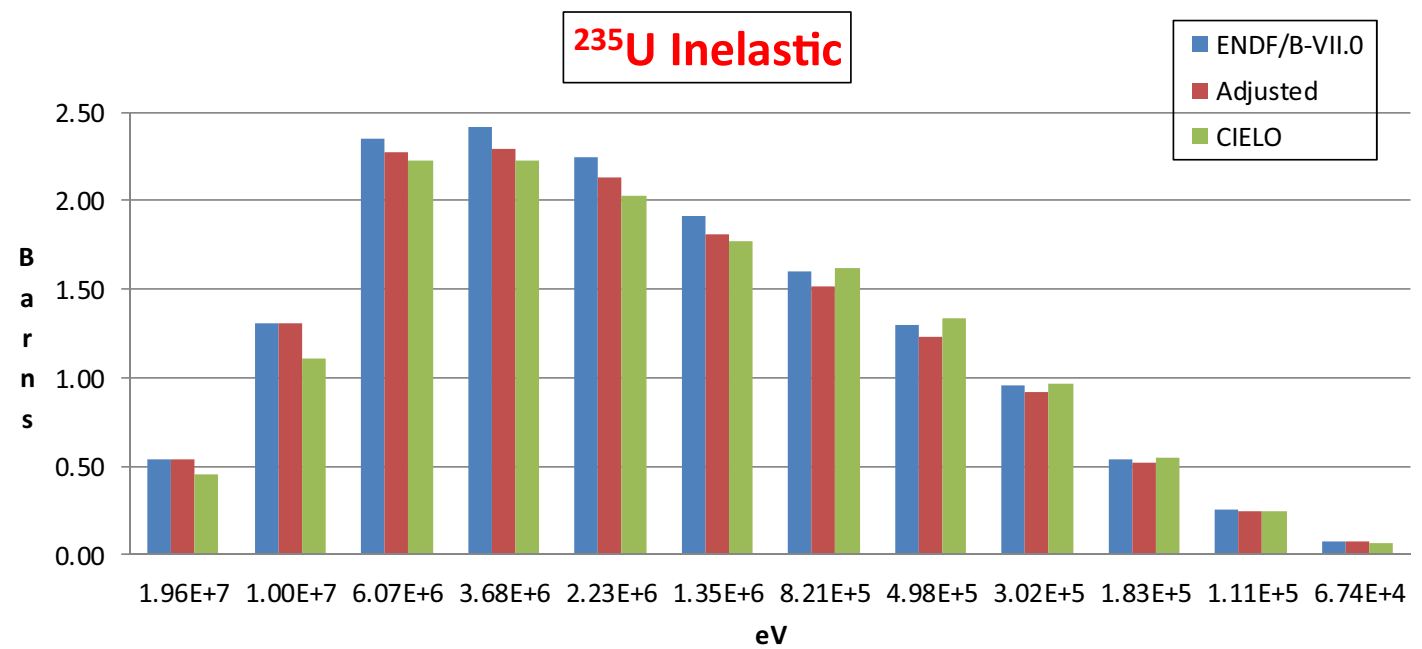

Figure 8. Examples of adjusted cross sections: ${ }^{235} \mathrm{U}$ inelastic.

\section{${ }^{235} U$ Fission Spectrum}

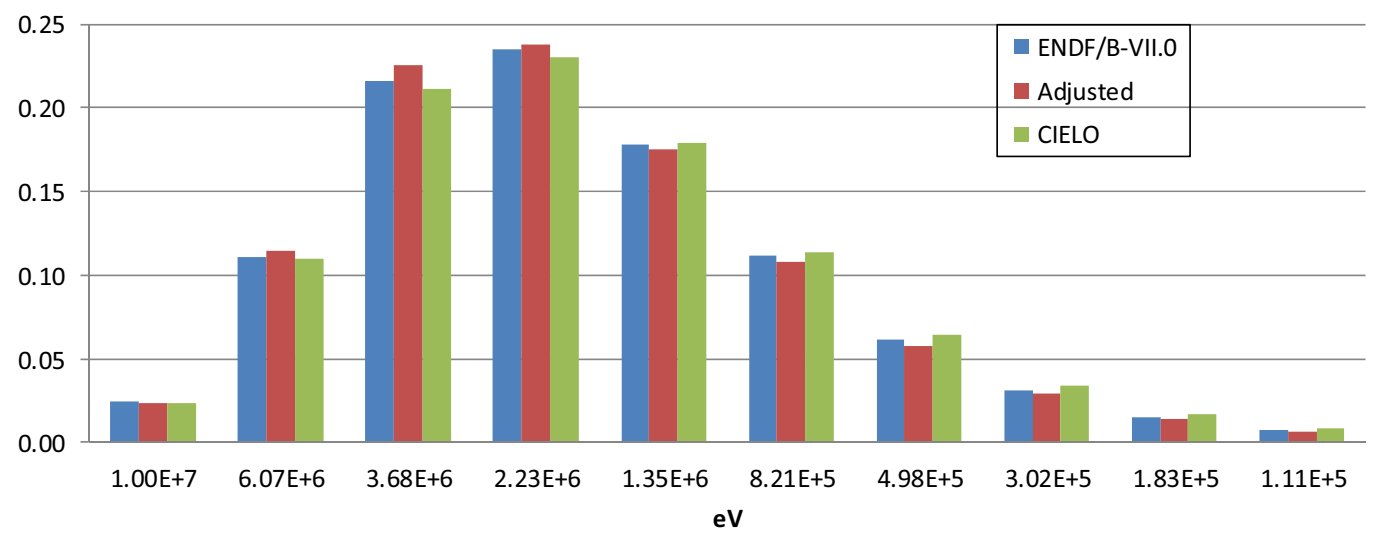

Figure 9. Examples of adjusted cross sections: ${ }^{235} \mathrm{U}$ fission spectrum.

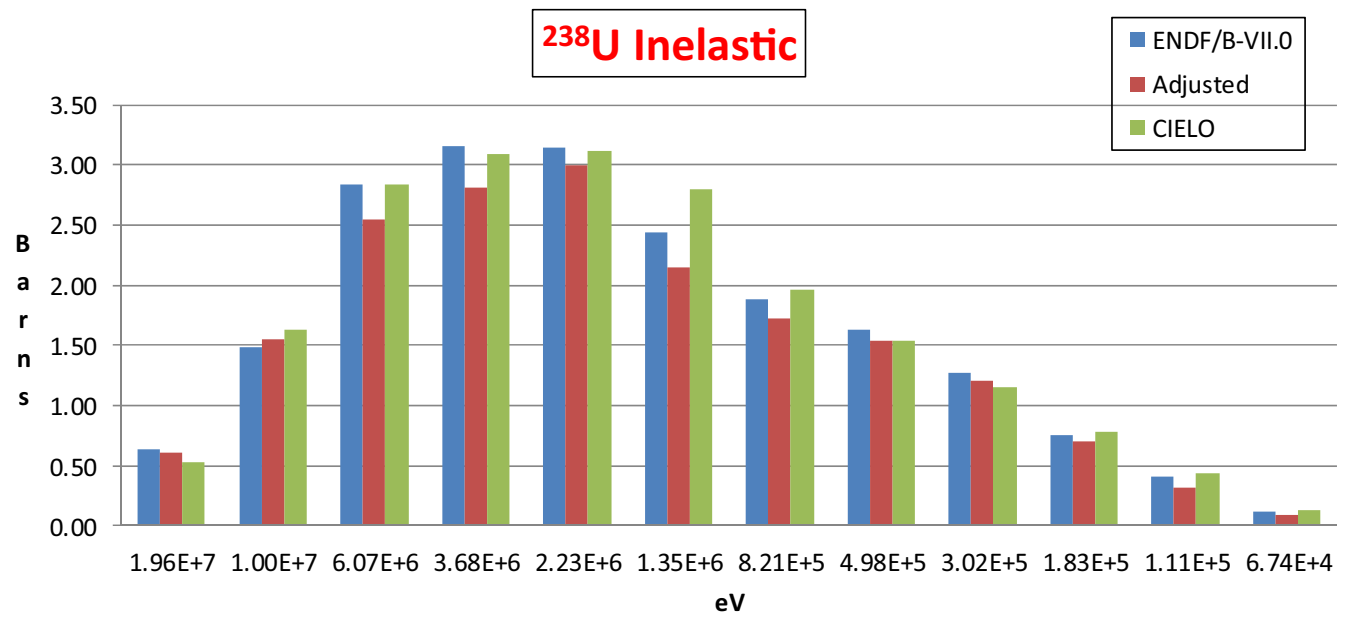

Figure 10. Examples of adjusted cross sections: ${ }^{238} \mathrm{U}$ inelastic. 


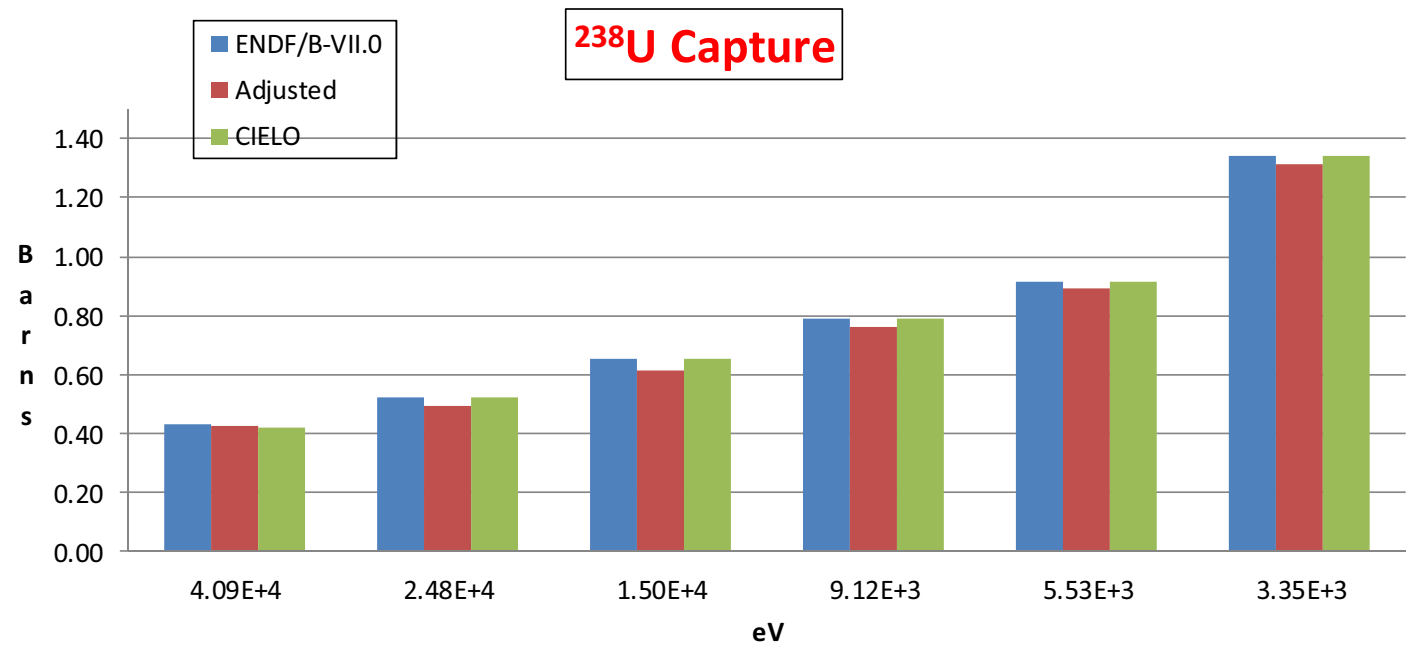

Figure 11. Examples of adjusted cross sections: ${ }^{238} \mathrm{U}$ capture.

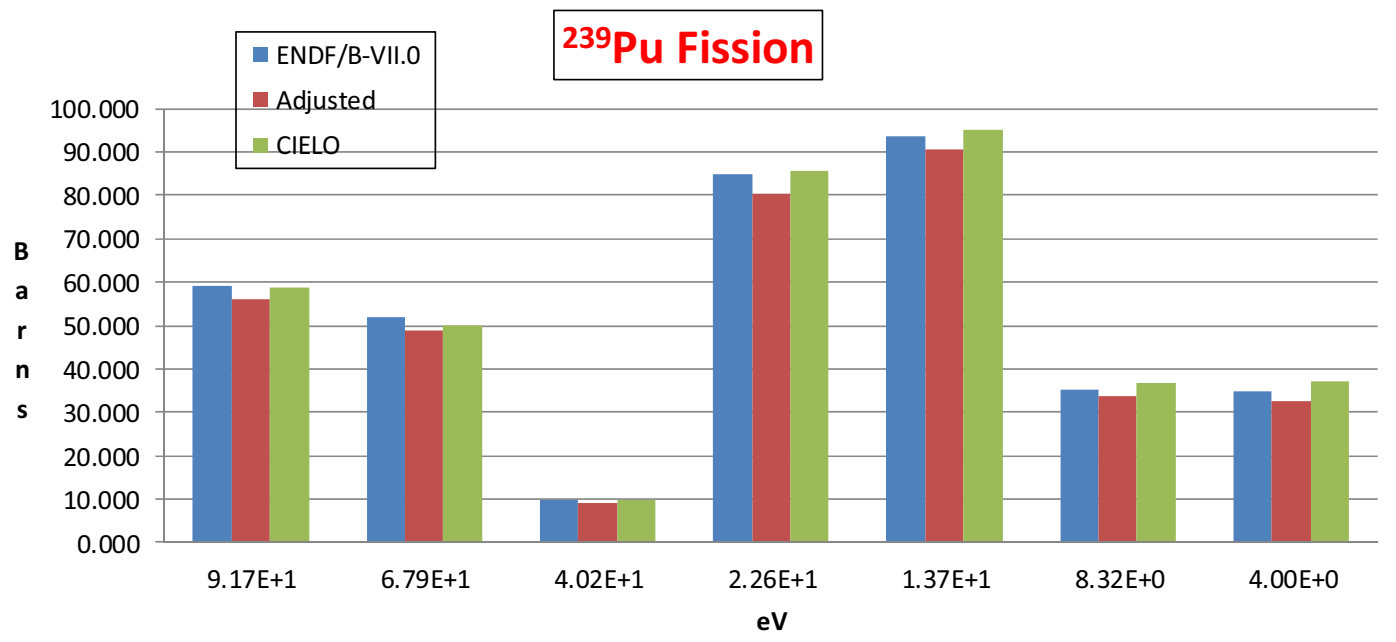

Figure 12. Examples of adjusted cross sections: ${ }^{239} \mathrm{Pu}$ fission.

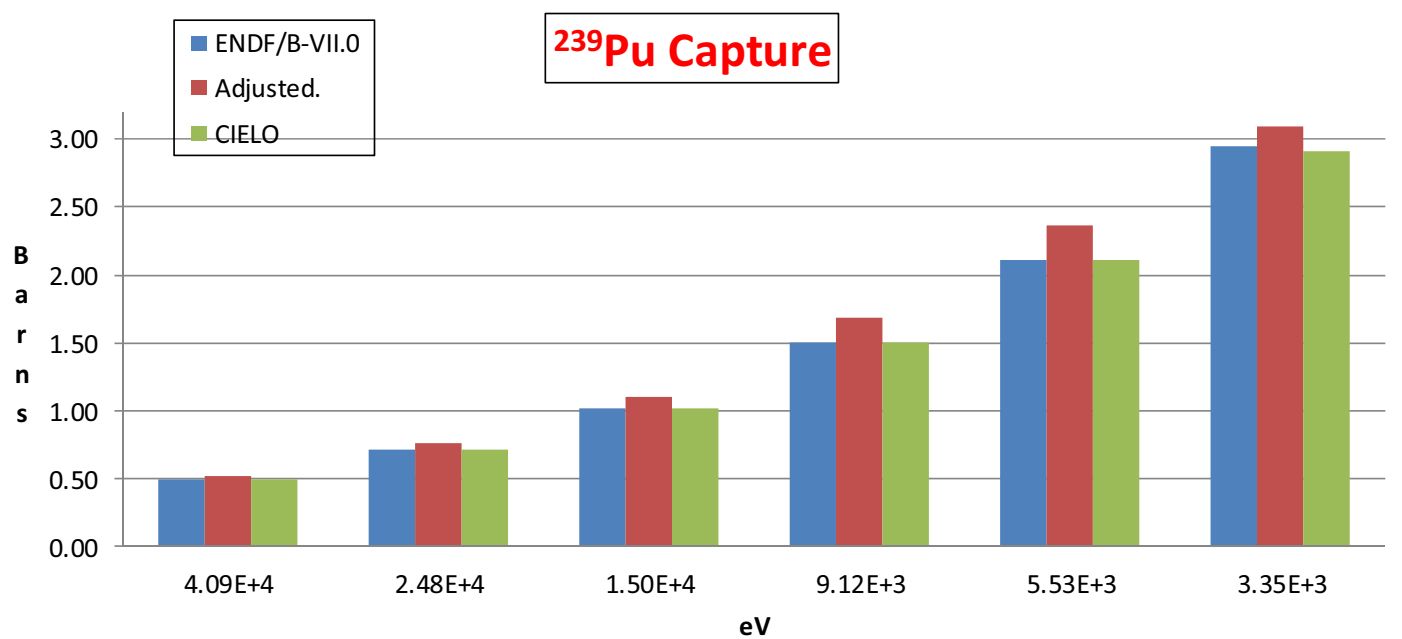

Figure 13. Examples of adjusted cross sections: ${ }^{239} \mathrm{Pu}$ capture. 


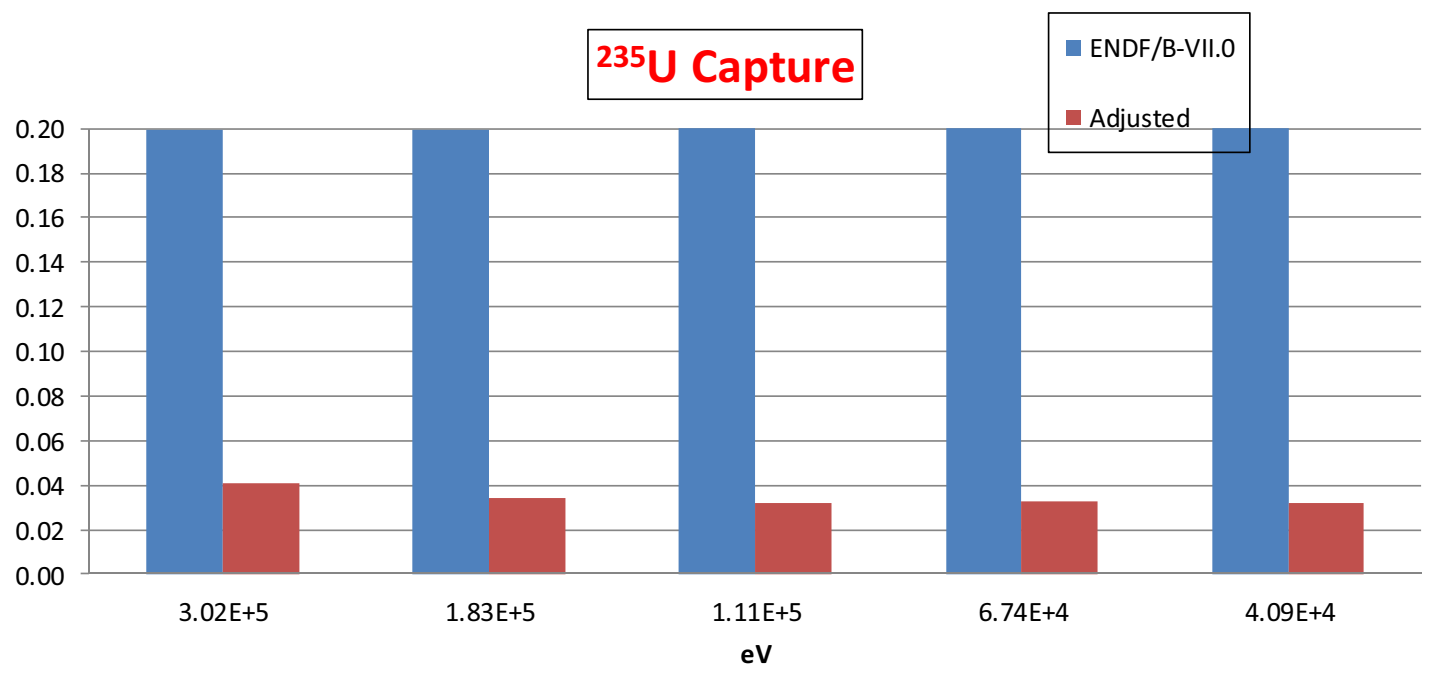

Figure 14. Standard deviations before and after adjustment: ${ }^{235} \mathrm{U}$ capture.

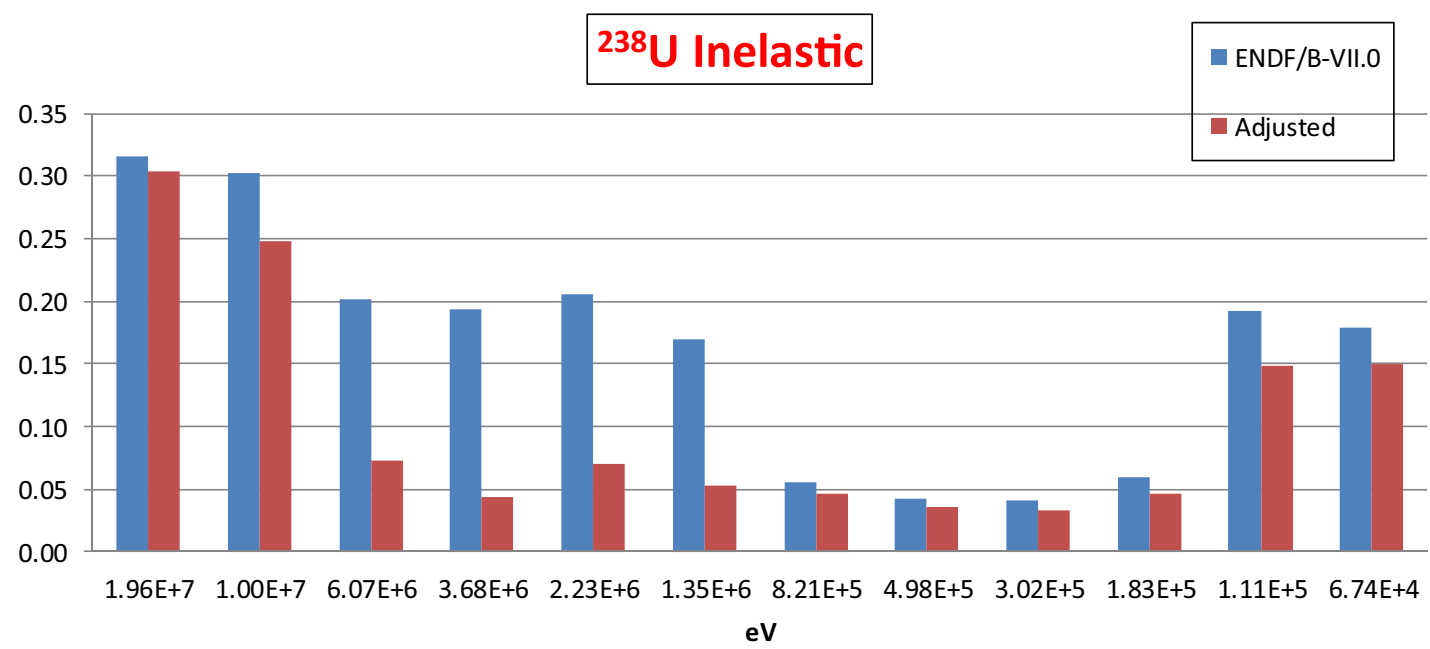

Figure 15. Standard deviations before and after adjustment: ${ }^{238} \mathrm{U}$ inelastic.

impact, as expected, the ${ }^{56} \mathrm{Fe}$ capture, elastic, and inelastic cross sections.

Finally, clear indications for the improvement of some FP capture cross sections $\left({ }^{105} \mathrm{Pd},{ }^{101} \mathrm{Ru},{ }^{151} \mathrm{Sm},{ }^{133} \mathrm{Cs}\right.$, ${ }^{153} \mathrm{Eu},{ }^{106} \mathrm{Pd}$, and ${ }^{95} \mathrm{Mo}$ ) have been pointed out.

\section{Conclusions}

An extended adjustment has been carried out adding "elementary type" of experiments often intended for specific reactions and energy ranges of the 5 isotopes of interest of the CIELO initiative, namely ${ }^{16} \mathrm{O},{ }^{56} \mathrm{Fe},{ }^{235} \mathrm{U}$, ${ }^{238} \mathrm{U}$, and ${ }^{239} \mathrm{Pu}$. Adjustments were also provided for a number of important FP isotopes.

The down selection of experiments (eliminating experiments probably affected by systematic errors or with conflicting $\mathrm{C} / \mathrm{E}$ values or also with unrealistically low uncertainties) together with the introduction of a significant new integral data base, has allowed to produce adjustments with an excellent level of statistical reliability.

Feedback has been provided for the 5 isotopes selected as priority in the CIELO initiative: some discrepancies are observed with the current proposed CIELO evaluations for some reactions of all the 5 isotopes. It is suggested to perform a further examination of the associated evaluations.

An important result of the present study is that major uncertainty reductions are observed for most isotopes.

As for possible future steps, especially in view of new method developments that are underway (e.g., PIA [12], continuous energy adjustment,), and most importantly for avoiding, as far as possible, compensations, it is very important that reliable and improved covariance data are provided by the evaluation community.

In particular there are still missing important data in covariance matrix data: some fission spectra, the $\mathrm{P}_{1}$ moment of elastic scattering for most isotopes, secondary energy distribution for inelastic cross sections (multigroup transfer matrix), cross correlations (reactions and isotopes), and delayed data (nubar and fission spectra).

In the future, finer energy grids and eigenvalue decomposition of the covariance matrix will be welcome. 
Finally, it is confirmed the essential role played by integral experiments, if the new criteria developed within the WPEC expert subgroups 33 and 39 for their selection are systematically applied.

This submitted manuscript was authored by a contractor of the U.S. Government under DOE Contract No. DE-AC0705 ID14517.

\section{References}

[1] "Uncertainty and Target Accuracy Assessment for Innovative Systems Using Recent Covariance Data Evaluations", International Evaluation Co-operation 26, NEA/WPEC-26, OECD/NEA, Paris (2008)

[2] M. Salvatores et al., "Methods and Issues for the Combined Use of Integral Experiments and Covariance Data: Results of a NEA International Collaborative Study," Nucl. Data Sheets 118, 38 (2014)

[3] "Methods and approaches to provide feedback from nuclear and covariance data adjustment for improvement of nuclear data files", International Evaluation Co-operation, NEA/WPEC-39, OECD/ NEA, ongoing

[4] M. Chadwick et al., "The CIELO Collaboration: Neutron Reactions on ${ }^{1} \mathrm{H},{ }^{16} \mathrm{O},{ }^{56} \mathrm{Fe},{ }^{235},{ }^{238} \mathrm{U}$, and ${ }^{239} \mathrm{Pu}$ ", Nucl. Data Sheets 118, 1-25 (2014)

[5] K. Fahrmann, D. Albert, H.U. Barz, K. Dietze, W. Hansen, D. Hedderich, G. Huttel, H. Krause, E. Lehmann, B. Osmera, W. Vogel, "Der Einsatz des Rossendorfer Rinzonenreaktors fur Untersuchungen zur Physik Schneller Reaktoren", Kernenergie 25(2), 464-471 (1982)
[6] M. Fukushima et al. "Fission Rate Ratios of FCA-IX Assemblies as Integral Experiment for Assessment of TRU's Fission Cross Sections" WONDER-2015 4th International Workshop on Nuclear Data Evaluation for Reactor applications, 2016

[7] G. Palmiotti et al., "Combined Use of Integral Experiments and Covariance Data," Nucl. Data Sheets 118, 596 (2014)

[8] G. Perret et al. Toward Reanalysis of the TightPitch HCLWR-PROTEUS Phase II Experiments, WONDER-2015 - 4th International Workshop on Nuclear Data Evaluation for Reactor applications, 2016

[9] G. Youinou, et al., "MANTRA: An Integral Reactor Physics Experiment to Infer Actinide The Neutron Capture Cross Sections Of Actinides And Fission Products In Fast And Epithermal Spectra", Nuclear Data Sheets 119, 169-172 (2014)

[10] I. Kodeli, G. Žerovnik, A. Milocco, Examples of Use of SINBAD Database for Nuclear Data and Code Validation, Proc. ICRS13-RPSD2016 Conf., Paris, Oct. 3-6, 2016. "Assessment of Existing Nuclear Data Adjustment Methodologies", International Evaluation Cooperation, Intermediate Report of WPEC Subgroup 33, NEA/NSC/WPEC/DOC (2010)429, OECD/NEA, Paris, 2011

[11] M.W. Herman et al., "COMMARA-2.0 Neutron Cross Section Covariance Library," Report BNL-94830-2011, Brookhaven National Laboratory (2011)

[12] G. Palmiotti, M. Salvatores, "PIA and REWIND: Two New Methodologies for Cross Section Adjustment", submitted to M\&C 2017, April 2017, Jeju, Korea 